\title{
Towards an emergent model of solitonic particles from non- trivial vacuum structure
}

\author{
Adam B. Gillard ${ }^{1, a}$ and Niels G. Gresnigt ${ }^{1}$ \\ ${ }^{1}$ Department of Mathematical Sciences, \\ Xi'an Jiaotong-Liverpool University, \\ 111 Ren'ai Road, Suzhou HET, Jiangsu, \\ China 215123
}

\begin{abstract}
We motivate and introduce what we refer to as the principles of Lie-stability and Hopf-stability and see what the physical theories must look like. Lie-stability is needed on the classical side and Hopf-stability is needed on the quantum side. We implement these two principles together with Liedeformations consistent with basic constraints on the classical kinematical variables to arrive at the form of a theory that identifies standard model fermions with quantum solitonic trefoil knotted flux tubes which emerge from a flux tube vacuum network. Moreover, twisted unknot fluxtubes form natural dark matter candidates.
\end{abstract}

\section{Introduction}

The goals of this paper are to motivate the need for both Lie and Hopf-type deformations in physics, define and explain the need for Lie and Hopf-stability, implement these broad but powerful physical principles, and see what the class of physical theories look like that result from satisfying these important principles.

We present our ideas in two stages. Firstly we look at physics from a classical viewpoint and discuss why we are forced to consider Lie-type deformations of underlying Lie-algebraic symmetries. Secondly we look at physics from a quantum viewpoint, in two stages. In the first stage we consider what the classical limit of a background independent fully emergent quantum theory should look like. In the second stage we use these considerations, enlightened by our previous classical level considerations, to arrive at a fully quantum theory of spacetime and matter. We sketch the motivational outline of the theory in this brief conceptual essay and leave the more lengthy details to be presented elsewhere.

Our model is built on two significant underlying assumptions.

1. There exist fundamental quantum states which define everything, including space and time (and hence gravity), and that all physical phenomena can be thought of as emergent

ae-mail: Adam.Gillard@xjtlu.edu.cn 
from the fundamental self-interacting quantum states.

This is essentially identical to the operational viewpoint of loop quantum gravity except in that case matter fields are thought of as being both additional and fundamentally different from the quantum gravitational fields. In our view, the matter field quantum states become additional when closed knotted flux tubes become free (i.e. emerge) from the spacetime and vacuum defining flux tube network.

2. The universally observed cosmological scale features of spacetime, i.e. the cosmological constant and the cosmic microwave background radiation, are intimately linked with the underlying structure of the physical background independent vacuum.

Loop quantum gravity operationally takes this viewpoint when it comes to the cosmological constant. ${ }^{1}$ We believe that perhaps we should be taking, additionally, the cosmic microwave background radiation into similar analogous consideration.

These assumptions together with our Lie and Hopf-algebraic stability considerations (which we discuss next and throughout the rest of this paper) leads to a physical model which is physically significantly different to the usual loop quantum gravity with cosmological constant and matter fields, which are purely thought of as being both physically distinct and additional to the quantum gravitational fields.

We need to define Lie-stability. If a Lie algebra $g_{0}$ with basis $\left\{X_{i}\right\}$ and bracket $\left[X_{i}, X_{j}\right]=$ $i f_{i j}^{k} X_{k}$ and a one parameter deformation of $g_{0}$ to $g_{t}$ defined by $\left[X_{i}, X_{j}\right]_{t} \equiv\left[X_{i}, X_{j}\right]_{0}+$ $\sum_{m=1}^{\infty} \phi_{m}\left(X_{i}, X_{j}\right) t^{m}$ with $g_{t}$ isomorphic to $g_{0}$, the Lie algebra is Lie-algebraically stable, i.e. Lie-stable $[2,3] .{ }^{2}$ Physically the Lie-stability of a classical symmetry algebra is saying that the theory is robust and free of fine tuning issues.

This is so because the structure constants in a Lie algebra of a physical theory often depend on physical constants. The numerical values of these physical constants must be determined experimentally and are therefore not known without some error. Lie-stability therefore provides insight into the validity of a physical theory or the need to generalize the theory. An unstable theory might be deformed until a stable theory is reached which is likely to be of wider validity than that of the original unstable theory.

The importance of Lie-stability in physical theories was first promoted by Mendes [3]. Since then, several others, most notably Chryssomalakos and Okon [4], and Ahluwalia [5] have similarly argued that the stability of a physically relevant Lie algebra should be considered a physical principle, on an equal footing with the principle of local gauge invariance, where, since each fiber of a vector bundle is isomorphic to $\mathbb{R}^{n}$ and a canonical isomorphism is absent, the laws of physics should not depend on any particular choice of local trivialization of any vector bundles.

The deformation parameters that arise in the stabilization of an algebra correspond to new invariant scales. These invariant scales constrain the permissible values that certain kinematic variables can take and therefore restricts the theory. We note however that the numerical values of these invariant scales are not obtained via algebraic considerations but must be determined in the laboratory. Lie-type deformations therefore provide a systematic method of introducing additional invariant scales. Lie-stability is both a physically sensible

\footnotetext{
${ }^{1}$ The Kodama state [1] is predominantly interpreted as a quantum gravitational ground state, which in the classical limit is identified with de Sitter spacetime for positive cosmological constant.

${ }^{2}$ The Lie algebra needs to be isomorphic under every Lie-deformation.
} 
requirement in the sense that it provides an indication of the robustness of a theory, as well as mathematically unambiguous in the sense that the stable algebra is unique up to isomorphism.

If a physical theory that is Lie-unstable has a domain of validity where the theory works quite well, this suggests that the theory is not necessarily wrong as such, but rather that it merely requires deformation to a hopefully true, Lie-stable physical theory. Deforming a Lie algebra is done in part by introducing a deformation parameter corresponding to some physically invariant scale. For example, if we constrain the speed kinematical variables $v^{i}$ with the physical constraint $0 \leq v^{i} \leq c<\infty$, we get a new type of length, $x_{0} \equiv c t$ which belongs to a four-dimensional manifold, the (non-gravitational) result being the usual special relativity with Minkowski spacetime isometry group $\mathbb{R}^{1,3} \rtimes S O(1,3)$, the associated Lie algebra of which, is still Lie-unstable. It is remarkable that imposing a physically realistic constraint on one of the kinematical variables produced a new theory with a much greater domain of validity, especially given that the quantum field theory of the standard model is constructed to respect the Poincare symmetries. It is natural then to ask whether there exist additional physically necessary constraints on the kinematical variables, and then to ask if the resulting Lie algebra is Lie-stable, which would then make it at least a possible viable candidate for the true classical limit of some true quantum theory of universal validity.

Finkelstein [6-13], showed that the standard model particles (as well as natural dark matter candidates), with their characteristic defining quantum electroweak and quantum chromodynamic quantum numbers, can be thought of as knotted quantum solitons corresponding to representations of the quantum group $S U_{q}(2) .^{3}$ Finkelstein's model is important for us because it presents important physical interpretations of the quantum groups $S L_{q}(2)$ and $S U_{q}(2)$, both quantum groups of which are important for our model. For this reason we include a brief but important appendix summarizing many key ideas in Finkelstein's work. We refer to a Hopf algebra with $q \neq 1$ as Hopf-stable, for reasons which will become clearer later.

\section{Classical physics and Lie-stability}

In classical Galilean relativity we have a configuration space $\mathcal{C}$ with position elements $\mathbf{x}$ on which there is a Euclidean metric which is preserved by the isometry group (ignoring the discrete symmetry transformations) $I S O(3) \equiv \mathbb{R}^{3} \rtimes S O(3)$. Moreover, phase space is $T^{*} \mathcal{C}$, speed $|\mathbf{v}| \equiv \frac{d|\mathbf{x}|}{d t}$ is an element of the coset space $I S O(3) / S O(3)$ and time $t$ is a free parameter. In this setting we have unconstrained kinematical variables, $0 \leq|\mathbf{x}|<\infty, 0 \leq|\mathbf{v}|<\infty$, $0 \leq t<\infty$. Similar statements hold for energy, momentum and mass. The theory is very successful within a relatively narrow domain of validity where the distances, times and speeds are not too "extreme." Moreover, if one or more of these variables starts tending in an extreme direction, the theory is violated. The interesting point is that the degree of violation smoothly increases. This suggests that the theory is simply incomplete in a certain sense rather than outright wrong. Special relativity can be interpreted as simply the result of imposing a physically realistic constraint on the kinematic variables, specifically,

\footnotetext{
${ }^{3} \mathrm{~A}$ quantum group is a quantum Hopf algebra, i.e. a bialgebra with the additional notion of antipode $[14,15]$. This is not a mathematical group. It can be thought of loosely as taking the symmetries which are present in a corresponding Lie-theory, incorporating them into the category of algebras, and then deforming with deformation parameter $q$ within the category of algebras. The category of Lie groups is rigid, i.e. not deformable, hence a need to consider the Hopf algebras. The deformation parameter $q$ provides the additional degree of freedom necessary to get away from considering pointlike particles to considering knotted quantum solitons. In the limit $q \rightarrow 1$, the degenerate case of pointlike particles is restored.
} 
a finite upper bound $c$ on the speeds such that now $0 \leq|\mathbf{v}| \leq c<\infty$. Here the space manifold has increased from three-dimensional to four-dimensional with a new distance $x_{0} \equiv$ ct made possible by the dimensionless deformation parameter $c$. Nature has such a constraint deformation parameter so the imposition of this constraint on the theory increased the now constrained theory's domain of validity. Moreover the quantization of this improved theory leads directly to the standard model of particle physics. A Lie-deformation has thus turned out to be quite revolutionary to physics. This theory, although improved, is still imperfect. In our Lie-stability paradigm it can immediately be recognized that the theory cannot be perfect because the Minkowski metric has an isometry group $\mathbb{R}^{1,3} \rtimes S O(1,3)$ which is Lieunstable. The problems of ultraviolet and infrared divergences hint at what the problem might be. The natural question to ask is whether there should exist additional constraints, $0<l_{p} \leq|\mathbf{x}| \leq l_{c}<\infty$ (the minimal length scale $l_{p}$ together with $c$ implying a minimum time $t_{p}$ such that $\left.0<t_{p} \equiv \frac{l_{p}}{c} \leq t<\infty\right)$. We consider physical motivation for $l_{p}$ and then consider the need for $l_{c}$.

Small distance scales require high energy to probe. There is a natural point at which the energy put into the system is sufficiently high to form a black hole so there should be a natural notion of a minimum length scale below which, no meaningful concept of external observers can be assigned. Moreover, Bronstein showed that localization to arbitrary precision is impossible in the presence of a gravitational field [16]. There exist physical grounds for taking the addition of a minimum nonzero length constraint seriously. Following the deformation procedure that took Galilean relativity to special relativity, deforming the Minkowski spacetime by imposing the additional kinematical constraint $0<l_{p} \leq|\mathbf{x}|$ gives a new natural length, $x_{4} \equiv l_{p}^{-1} A$ where $A$ is some parameter with dimensions of area. The resulting spacetime has metric $d s^{2}=c^{2} d t^{2}+l_{p}^{-2} d A^{2}-d \mathbf{x}^{2}$ which is preserved by the isometry group $\mathbb{R}^{2,3} \rtimes S O(2,3)$ which again has a Lie-unstable algebra. The introduction of both $c$ and $l_{p}$ naturally also implicitly introduces a minimal time $t_{p} \leq t$. We could develop this theory on a now five-dimensional manifold further but in our view it still would not be ultimately correct because the symmetry Lie algebra is still Lie-unstable.

We are not aware of any need for a nonzero minimum speed or a maximum time so the only obvious further physical constraint on the kinematical variables is to impose a maximum length scale $l_{c}$. It is natural to do this because such a scale is related to a positive cosmological constant $\Lambda \approx l_{c}^{-1 / 2}$. Such a constant has been observed so we have a natural additional length $x_{5} \equiv l_{c} \phi$ where $\phi$ is some dimensionless parameter, the interpretation of which we consider later. The resulting metric is then six-dimensional. This kinimatical space (which contains what we will call kinematical timelike, spacelike and lightlike intervals) is not physical however. Physical space can only be defined on the five-dimensional hypersurface ${ }^{5}$

$$
-\left(\frac{d s}{d \phi}\right)^{2}+c^{2}\left(\frac{d t}{d \phi}\right)^{2}+l_{p}^{-2}\left(\frac{d A}{d \phi}\right)^{2}-\left(\frac{d \mathbf{x}}{d \phi}\right)^{2}=l_{c}^{2} .
$$

We see immediately that the induced metric for timelike intervals has isometry group $S O(2,4)$ corresponding to a type of five-dimensional anti-de Sitter space $A d S_{5}$ and isomorphic to the

\footnotetext{
${ }^{4}$ moreover, in loop quantum gravity, classically large scale infrared divergences in the transition amplitudes are tamed by $q$-deforming the theory, which, in the classical limit yields Einsteins equations with positive cosmological constant [17]. This in turn then implies a related cosmological length scale $l_{c}$. This demonstrates a need for Hopf-stability.

${ }^{5}$ if we "divide through" by the dimensionless quantity $d \phi^{2}$ we get a five-dimensional hypersurface defined by quantities which are still lengths. Moreover, this space is hyperbolic which, being curved, cuts out the abelian translation group.
} 
conformal group. This space has an asymptotic boundary on which one has a natural notion of a 1+3-dimensional conformal field theory $C F T_{4}$ by the $A d S / C F T$ correspondence. Also, for spacelike intervals we have a five-dimensional space with induced metric having isometry group $S O(3,3)$. For lightlike intervals we have a space with induced metric having isometry group $S O(2,3)$ corresponding to a type of four-dimensional anti-de Sitter space $A d S_{4}$. This has an asymptotic boundary on which there exists a natural notion of a 1+2-dimensional conformal field theory $\mathrm{CFT}_{3}$. The Lie algebras of these isometry groups are all Lie-stable so we have arrived at something which feasibly could be the classical limit of some true quantum gravitational ground state in some universally valid quantum theory. A natural question to ask now is: is the physical space five-dimensional, or do there exist any additional constraints? By observation we appear to live in a 1+3-dimensional universe so one would expect there to exist one additional constraint. The existence of a minimal length scale and a maximum length scale introduces a natural notion of a radius of curvature and a natural notion of a minimum angular resolution $\phi_{p}=l_{p} l_{c}^{-1}$. The existence of a universal radius of curvature, say $R$, would suggest that $\frac{d|\mathbf{x}|}{d \phi}=R=$ constant. This defines a two-dimensional space $S^{2}$ with induced metric having isometry group $S O(3)$. But from Eqn. (1) this implies that

$$
-\left(\frac{d s}{d \phi}\right)^{2}+c^{2}\left(\frac{d t}{d \phi}\right)^{2}+l_{p}^{-2}\left(\frac{d A}{d \phi}\right)^{2}=\text { constant } \quad\left(=l_{c}^{2}+R^{2}\right) .
$$

For timelike intervals this defines a two-dimensional constraint surface with induced metric having isometry group $S O(2,1)$. For spacelike intervals this defines a two-dimensional constraint surface with induced metric having isometry group $S O(3,0)$. For lightlike intervals this defines a one-dimensional constraint surface with induced metric with isometry group $S O(2,0)$. Putting the two two-dimensional spaces together, for timelike intervals, we have a direct product space $A d S_{2} \times S^{2}$ which is a four-dimensional spacetime composed of two twodimensional bits; a 1+1-dimensional space and a two-dimensional space. We recognize this from general relativity as the Bertotti-Robinson spacetime $[18,19]$. The space corresponds to the spacetime solution to the Einstein-Maxwell equations with positive cosmological constant where the energy-momentum tensor $T_{\mu \nu}$ is composed of a cosmological constant term and two terms corresponding to electromagnetism. If this were to be physically realistic we would expect the universe to be full of electromagnetic radiation. At this point we note that universal cosmological background radiation has been observed and its existence is universal just as the cosmological constant is universal. For the spacelike case the physical hypersurface we have from Eqn. (2) a two-dimensional space with induced metric having isometry group $S O(3)$, so the total four-dimensional space is $S^{2} \times S^{2}$ with induced metric having isometry group $S O(3,0) \times S O(0,3)$. Finally, for the lightlike case we have $S^{1} \times S^{2}$ with induced metric having isometry group $S O(2,0) \times S O(0,3)$.

We conclude that $A d S_{2} \times S^{2}, S^{2} \times S^{2}$ and $S^{1} \times S^{2}$ constitute complementary viable candidates for a target classical limit that a true quantum theory would reproduce as a quantum gravitational ground state (i.e. in the absence of matter).

In the standard model of particle physics the underlying spacetime symmetry group is the Poincaré group $\mathcal{P}=\mathbb{R}^{1,3} \rtimes S O(1,3)$ of special relativity. The transition to quantum field theory from classical special relativity can be obtained by taking the infinite-dimensional unitary representations $U(\Lambda, a)$ of this isometry group of the fixed background structure with fixed Minkowski metric, act on a Hilbert space $\mathcal{P}: H \rightarrow H$ and invoke the quantum mechanical axioms to interpret the states in Hilbert space. Then adjoin the internal symmetry groups to get $\mathcal{P} \times S U(3)_{c} \times S U(2)_{L} \times U(1)_{Y}: H \rightarrow H$ where the details of the internal 
symmetry group are apparently taken from experiment with no obvious theoretical origins. This theory is non-gravitational since there is no spacetime curvature dynamics in the theory. If we take the same approach here, also thought of as a non-gravitational theory for the time being, then for our timelike case we would write down $S O(2,1) \times S O(3): H \rightarrow H$ by specifying the infinite-dimensional unitary representations (this group is also non-compact so its nontrivial unitary representations are infinite-dimensional). In practice, we would actually write $S L(2, R) \times S U(2)$ since $s l(2) \cong s o(2,1)$ and $S U(2)$ is the double cover for $S O(3)$ (the former having representations while the latter only having projective representations) so we have $S L(2) \times S U(2): H \rightarrow H$. However, thinking of our symmetry groups in this way leaves gravity out of the picture, whereas a full quantum theory however must take into account quantum gravity. For the other cases we would have respectively $S U(2) \times S U(2)$ : $H \rightarrow H$ and $U(1) \times S U(2): H \rightarrow H$. This lightlike case is interesting for several reasons. Firstly, it is mathematically the same as the electroweak gauge group of the standard model. Secondly, if we take the universal enveloping algebra $\mathcal{U}(u(1) \oplus s u(2))$ and $q$-deform to the quantum group $\mathcal{U}_{q}(u(1) \oplus s u(2))$ this is isomorphic to $\mathcal{U}_{q}(g l(2))$ which is also isomorphic to the braided matrices $B M_{q}(2)$ which in turn is isomorphic to the quantum Minkowski space $\mathbb{R}_{q}^{1,3}$. These isomorphisms themselves which only hold under $q \neq 1$ were discovered by Majid [20] and constitute powerful additional reasons for considering Hopf-deformations and braided geometry.

With these things in mind we now turn our attention to background and metric independent quantum theory.

\section{Quantum gravity and Hopf-stability}

In this section we take a more bottom-up approach to considering what any potential fully quantum theory needs, and then we harmonize the observations with those of the previous section.

Since the spacetime metric is the classical gravitational field, a quantum theory of spacetime which gives the classical structures in the classical limit must be background independent and hence also metric independent. The fundamental physical fields of nature should then be described by forms. These physical fields will have characteristic defining internal symmetries so they will be $G$-valued forms for some appropriate group $G$. Actually, they really need to be $G_{q}$-valued forms where $G_{q}$ is the corresponding quantum group. ${ }^{6}$ That is to say, the symmetries need to be Hopf-stable, i.e. there should be a principle of Hopf-stability, analogous with classical physics where we need a principle of Lie-stability. We take a few sentences to motivate this. Firstly we motivate wanting Hopf algebras instead of Lie groups and then we motivate wanting $q$-deformed Hopf algebras, i.e. Hopf-stable algebras, i.e. quantum Hopf algebras.

In ordinary classical physics the physical states are defined in phase space $\mathbb{R}^{2 n}$ with configuration space $\mathbb{R}^{n}$. In ordinary quantum mechanics in contrast, information about a physical state is encoded in the wavefunction $\psi: \mathbb{R}^{n} \rightarrow \mathbb{C}$. Furthermore, commuting position observables $x_{i}: \mathbb{R}^{n} \rightarrow \mathbb{C}$ act on wavefunctions in a multiplicative way. Geometry in ordinary quantum mechanics is described by the algebra $\mathcal{A}\left(\mathbb{R}^{n}\right)$ of functions over a space $\mathbb{R}^{n}$ rather than being described directly by the space $\mathbb{R}^{n}$ itself as is the case in ordinary classical physics.

\footnotetext{
${ }^{6}$ We were slightly sloppy here in saying $G$-valued rather than $g$-valued for the corresponding Lie algebra $g$. However, in either case, under $q$-deformation we get a quantum group and the two cases are dual to each other.
} 
Now suppose in classical physics there is some group $G$ of symmetries. We would incorporate this into the classical theory by writing $G \otimes \mathbb{R}^{n} \rightarrow \mathbb{R}^{n}$. In the quantum theory, in order to be mathematically internally consistent, instead of having $G$ directly, we should have the algebra $\mathcal{A}(G)$ of functions over $G$. It follows then that if we have an action $\phi$ of a group $G$ on the space $\mathbb{R}^{n}$ in the classical theory, then in the corresponding quantum theory $\mathcal{A}(G) \otimes \mathcal{A}\left(\mathbb{R}^{n}\right) \stackrel{\phi^{*}}{\longleftarrow} \mathcal{A}\left(\mathbb{R}^{n}\right)$ where $\phi^{*}$ is the pull-back and is a homomorphism of algebras and is called the coaction map. ${ }^{7}$ The axioms of the group under the action are translated into axioms of the algebra under the coaction. The group has structure maps; multiplication, unit and inverse. These are translated respectively into comultiplication, counit and antipode. An algebra with this additional co-structure is a hopf algebra. In general there are two dual quantum Hopf algebras $G_{q}$ and $\mathcal{U}_{q}(g)$, where $g$ is the Lie algebra of the Lie group $G$ and $\mathcal{U}$ denotes the universal enveloping algebra. When $q=1$ the usual group structures are restored.

Here is a key point. In classical Newtonian physics we have a Lie-unstable algebra due to the presence of an abelian translation group conjugate to a commutative spacetime. When $q=1$ we have Hopf algebras which are commutative and cocommutative. Our principle of emergence (i.e. everything physical is background independent and has to in a sense be "internally defined") naturally dictates that the physical fields must themselves be elements of the Hopf algebra. To get non-trivial fields the elements $T \in \mathcal{A}(G)$ must be non-cocommutative and this requires that the Hopf algebra be $q$-deformed. We refer to this as a Hopf-stability principle which is analogously to the quantum theory what the Lie-stability principle is to the classical theory.

We expect then, that our fully quantum theory must be of the form $G_{q}: H \rightarrow H$ where $H$ denotes the physical Hilbert space of physical states. The challenge then becomes to identify the correct $G_{q}$ and then elucidate all of the mathematical details, along with associated physical interpretation. If the theory is good, known observations will be explainable within the mathematical structure. The remainder of the mathematical structure would then dictate the theory's predictive power. Presumably if such a programme failed, a true theory of everything would have to be based on a different set of principles rather than quantum principles with the quantum principles emerging from the other set.

To help us determine what the correct quantum group $G_{q}$ should be, we make some observations from standard loop quantum gravity. An underlying assumption in the usual theory is that the classical limit of the quantum gravitational ground state should be flat Minkowski spacetime. The fundamental variables in the spin network representation are then Lorentzian, and on a 4-simplex, given by $U_{e}=P e^{\int_{e} \omega} \in S L(2, \mathbb{C})$ which discretizes the Lorentzian connection $\omega$ and $B_{f}=\int_{t_{f}} B \in \operatorname{sl}(2, \mathbb{C})$ ([17]) which discretizes the (complexvalued) frame field $e: \Sigma \times \mathbb{C}^{4} \rightarrow \mathbb{C} T M$. A problem is that when one tries to do quantum kinematics, i.e. calculate the transition amplitudes, the integrals are infrared divergent; infrared in the sense that the divergence comes about because of problems in the large scale (i.e. quantum gravitational high spin) structure. The standard fix is to $q$-deform the theory [21-24]. The classical limit of this $q$-deformed theory does not give flat Minkowski spacetime however. It gives a spacetime differing from Minkowski spacetime by the presence of a positive cosmological constant. This is an example of a theory which has problems when Hopf-unstable which disappear if one moves to a Hopf-stable theory. In the connection representation a viable quantum gravitational state is the Kodama state [1]. This state seems to have a natural interpretation of being a ground state, and it again crucially depends on a

\footnotetext{
${ }^{7}$ See $[14,15]$ for more mathematical details.
} 
nonzero cosmological constant. If positive, the ground state in the classical limit corresponds to de Sitter spacetime. This Kodama state has some potential problems however [25]. ${ }^{8}$ In that same work Randono introduces a generalized set of Kodama states which solves some of the problems. The work involves trying to make a properly quantum de Sitter based quantum gravity. Interestingly, on p.111 Randono states:

The true constraint algebra is likely to be a deformation of the de Sitter Lie algebra. The deformation itself contains the local degrees of freedom of general relativity. Since the action possesses an exact de Sitter symmetry on a very large portion of the phase space (anywhere $t=0$ ), the constraint algebra is likely to reduce to the de Sitter algebra on a large portion of the constraint manifold.

Summarizing the last few observations: Ordinary loop quantum gravity in the spin network representation showed a need for fixing to properly regulate quantum observables. The fix was to $q$-deform; the classical result being the inclusion of a positive cosmological constant. If one includes such a cosmological constant and looks at loop quantum gravity in the connection representation one finds the Kodama state. However this too is not (necessarily) problem free. A suggested fix is to $q$-deform the de Sitter based loop quantum gravity theory.

We propose that the classical limit of a true quantum gravitational ground state should look like $A d S_{2} \times S^{2}$ spacetime for our timelike case, $S^{2} \times S^{2}$ spacetime for our spacelike case and $S^{1} \times S^{2}$ spacetime for our lightlike case. This results from our Lie-stability arguments together with the corresponding twin problem of making sure the kinematical variables are constrained and constrained in the way relevant to nature. At the quantum level however, we have argued that we want a quantum Hopf algebra so we want physical fields in general based on $S L_{q_{1}}(2) \times S U_{q_{2}}(2)$ for the timelike case. To tighten up what our theory should look like, we note that in the un- $q$-deformed case, we have a direct product of two two-dimensional spaces. The fact that the fundamental spaces are two-dimensional gives rise to a natural notion of $q$-deformations and also braiding, anyonic structures and quantum flux tubes [26], so an integrated internally consistent picture is starting to emerge. One question which remains is what the space is that the $q$-deformed Hopf algebra $S L_{q_{1}}(2) \times S U_{q_{2}}(2)$ acts on. It turns out ([20]) that braided spaces have quantum groups as their natural symmetries. Thus we want a braided version $B\left(A d S_{2}\right) \times B\left(S^{2}\right)$ of $A d S_{2} \times S^{2}$ upon which $S L_{q_{1}}(2) \times S U_{q_{2}}(2)$ acts. Here $x$ signifies that the tensor product between algebras has been deformed rather than the algebras themselves. This allows all relevant mathematical structures to be deformed in an internally consistent way $[15,20]$. Finally, for correct braid statistics normalization under braided co-addition the quantum group must be adjoined with a dilatation operator [20].

\section{Conclusion and a possible physical interpretation}

We have explored the consequences of both imposing the principles of Lie-algebraic and Hopfalgebraic stability, and imposing a more complete physically realistic set of constraints on the kinematical variables. On the classical Lie-stability side we arrived at three unphysical "kinematical" five-dimensional hypersurfaces distinguished by whether the metric on the parent six-dimensional space was describing a timelike interval, a spacelike interval or a lightlike interval. We also explored the consequences of a further restriction, $\frac{d|\mathbf{x}|}{d \phi}=R$, which resulted

\footnotetext{
${ }^{8}$ e.g. non-renormalizability issues, negative energies, CPT violation, non-invariance under large gauge transformations.
} 
in three product space cases, $\operatorname{Ad} S_{2} \times S^{2}, S^{2} \times S^{2}$ and $S^{1} \times S^{2}$ corresponding to the timelike case, the spacelike case and the lightlike case respectively. The respective symmetry groups are $S O(2,1) \times S U(2), S U(2) \times S U(2)$ and $U(1) \times S U(2)$. These Lie-stable spaces do not have an abelian translation group. The corresponding quantum theory should not have commuting coordinates either, so the corresponding commutative and cocommutative quantum Hopf algebras needed to be Hopf-stabilized (in other words, $q$-deformed). Hence our quantum symmetry groups are respectively $S L_{q_{1}} \times S U_{q_{2}}(2), S U_{q_{1}}(2) \underline{\times} S U_{q_{2}}(2)$ and $U_{q_{1}}(1) \underline{X} S U_{q_{2}}(2)$ for the timelike, spacelike and lightlike cases. The quantum spaces that these quantum groups (along with the adjoined dilatation operator needed for correct braid statistics normalization being preserved under braided co-addition) act on must be braided versions $B\left(A d S_{2} \times S^{2}\right)$, $B\left(S^{2} \underline{\times} S^{2}\right)$ and $B\left(S^{1} \times S^{2}\right)$ of the above mentioned classical spacetimes. In the standard model the matter particles are characterized by unitary irreducible representations of the (Poincaré) spacetime symmetry group (adjoined together with quantum numbers associated with the internal symmetry quantum electroweak and quantum chromodynamics gauge groups). Keeping the same general notion of compatibility with the physical vacuum, it is natural to demand that matter be characterized by representations of these above quantum groups.

We have seen from Finkelstein's work (see the appendix) that the standard model spin- $\frac{1}{2}$ fermions and spin-1 electroweak vector bosons can naturally be described by the representations of $S U_{q}(2)$ corresponding to quantum trefoil knots and quantum ditrefoil knots respectively. ${ }^{9}$ Moreover, the simpler, twisted unknots (which Finkelstein refers to also as preons) are natural dark matter candidates, and by virtue of them being more fundamental than the quantum trefoil and ditrefoil knots, would naturally be expected to be in higher abundance which is consistent with the observation that there must be more dark matter in the universe than standard model matter.

In our theory based on the fairly minimal and natural physical requirements of Lie and Hopf-stability together with a more complete and realistic set of physical constraints on the classical kinematical variables, both the physical vacuum and matter (both standard model and dark) are all in some deep way quantum gravitationally connected via the quantum group $S L_{q}(2)$ Hopf algebra of knotted flux tubes and its quantum subgroup $S U_{q}(2)$ Hopf algebra of oriented knotted flux tubes. It will surely be of great interest to elucidate the mathematical details, identify the parts of the theory that are predictive in nature, and figure out ways of testing those predictions to either verify or falsify the theory. Since the expansion of this theory is largely mathematical in nature with only a small number of physical axioms, any predictions should be well-defined and hence testable in principle, making the theory concretely falsifiable.

\section{Appendix: Finkelstein's knotted $S L_{q}(2)$-based extension of the standard model}

We briefly review the representations of $S L_{q}(2)$ and their restriction to $S U_{q}(2)$. The elements $L$ of the classical group $S L(2)$ satisfy

$$
\epsilon_{i j} L_{i m} L_{j n}=\epsilon_{m n} \operatorname{det} L=\epsilon_{m n}, \quad \longrightarrow \quad L^{t} \epsilon L=\epsilon .
$$

Here $L$ and $\epsilon$ take the forms

$$
L=\left(\begin{array}{ll}
a & b \\
c & d
\end{array}\right), \quad \epsilon=\left(\begin{array}{cc}
0 & 1 \\
-1 & 0
\end{array}\right) .
$$

\footnotetext{
${ }^{9}$ The case of the photon and $Z^{0}$ are slightly more complicated.
} 
To $q$-deform to $S L_{q}(2)$ we wish to preserve the essential characteristic that $\operatorname{det} \epsilon=1$. For later notational convenience we define $q_{1}=q^{-1}$ and deform the matrix $\epsilon$ in the obvious way:

$$
\epsilon_{q}=\left(\begin{array}{cc}
0 & q_{1}^{\frac{1}{2}} \\
-q^{\frac{1}{2}} & 0
\end{array}\right)
$$

We now want matrices $L_{q} \in S L_{q}(2)$ of the form

$$
L_{q}=\left(\begin{array}{ll}
a & b \\
c & d
\end{array}\right)
$$

such that

$$
L_{q}^{t} \epsilon_{q} L_{q}=\epsilon_{q}
$$

Defining the $q$-commutator $[A, B]_{q}=A B-q B A$ we see that after an explicit calculation based on Eqn.(7) the algebra that the elements of $L_{q}$ must satisfy is

$$
\begin{array}{r}
{[a, b]_{q}=0, \quad[a, c]_{q}=0, \quad[b, c]=0, \quad[b, d]_{q}=0, \quad[c, d]_{q}=0,} \\
a d-q b c=1, \quad d a-q_{1} b c \equiv \operatorname{det}_{q} L=1 .
\end{array}
$$

This marks a significant shift from the usual representation theory of classical Lie groups where the matrix elements are commutative numbers, whereas here they are noncommutative operators. The $q$-binomial theorem is required in order to specify the representations of $S L_{q}(2)$. We have the following definitions:

$$
\begin{aligned}
& (a+b)^{n}=\sum_{s}^{n}\left\langle\begin{array}{c}
n \\
s
\end{array}\right\rangle_{q} b^{s} a^{n-s}, \quad(a+b)^{n}=\sum_{s}^{n}\left\langle\begin{array}{c}
n \\
s
\end{array}\right\rangle_{q_{1}} a^{s} b^{n-s}, \\
& \left\langle\begin{array}{c}
n \\
s
\end{array}\right\rangle_{q}=\frac{\langle n\rangle_{q} !}{\langle n-s\rangle_{q} !\langle s\rangle_{q} !}, \quad\langle n\rangle_{q}=\frac{q^{n}-1}{q-1} .
\end{aligned}
$$

We work in the monomial basis where for $\left[x_{1}, x_{2}\right]_{q}=0, n_{ \pm}=j \pm m, N_{m}^{j}=\left[\left\langle n_{+}\right\rangle_{q_{1}} !\left\langle n_{-}\right\rangle_{q_{1}} !\right]^{\frac{-1}{2}}$ and $\psi_{m}^{j} \equiv N_{m}^{j} x_{1}^{n_{+}} x_{2}^{n_{-}}$. Taking $\mathbf{x}^{t}=\left[x_{1}, x_{2}\right]$, we write $\mathbf{x}^{\prime} \equiv L_{q} \mathbf{x}$. On the monomial basis $\psi_{m}^{j} \equiv N_{m}^{j} x^{n_{+}} x^{n_{-}}$, assuming that the $x_{i}$ commute with $a, b, c, d$, this transformation takes the form

$$
\psi_{m}^{j^{\prime}}=N_{m}^{j}\left(a x_{1}+b x_{2}\right)^{n_{+}}\left(c x_{1}+d x_{2}\right)^{n_{-}}
$$

and by use of the $q$-binomial thorem we directly obtain

$$
\psi_{m}^{j^{\prime}}=N_{m}^{j} \sum_{s}^{n_{+}} \sum_{t}^{n_{-}}\left\langle\begin{array}{c}
n_{+} \\
s
\end{array}\right\rangle_{q_{1}}\left\langle\begin{array}{c}
n_{-} \\
t
\end{array}\right\rangle_{q_{1}} a^{s} b^{n_{+}-s} c^{t} d^{n_{-}-t} x_{1}^{s+t} x_{2}^{n_{+}+n_{-}-s-t} .
$$

By defining $n_{ \pm}^{\prime}=j \pm m^{\prime}$ we make use of the Kroneckar delta $\delta\left(s+t, n_{+}^{\prime}\right)$ to write

$$
\begin{aligned}
\psi_{m}^{j^{\prime}} & =\sum_{s, t}\left(\frac{N_{m}^{j}}{N_{m^{\prime}}^{j}}\right) \delta\left(s+t, n_{+}^{\prime}\right)\left\langle\begin{array}{c}
n_{+} \\
s
\end{array}\right\rangle_{q_{1}}\left\langle\begin{array}{c}
n_{-} \\
t
\end{array}\right\rangle_{q_{1}} a^{s} b^{n_{+}-s} c^{t} d^{n_{-}-t}\left(N_{m^{\prime}}^{j} x_{1}^{n_{+}^{\prime}} x_{2}^{n_{-}^{\prime}}\right) \\
& =\sum_{m^{\prime}} D_{m m^{\prime}}^{j} \psi_{m^{\prime}}^{j}
\end{aligned}
$$


where

$$
D_{m m^{\prime}}^{j}(q \mid a, b, c, d) \equiv \sum_{s, t}\left(\frac{N_{m}^{j}}{N_{m^{\prime}}^{j}}\right) \delta\left(s+t, n_{+}^{\prime}\right)\left\langle\begin{array}{c}
n_{+} \\
s
\end{array}\right\rangle_{q_{1}}\left\langle\begin{array}{c}
n_{-} \\
t
\end{array}\right\rangle_{q_{1}} a^{s} b^{n_{+}-s} c^{t} d^{n_{-}-t}
$$

The $S U_{q}(2)$ representations are obtained by the above $S L_{q}(2)$ representations by setting $d=\bar{a}$ and $c=-q_{1} \bar{b}$. For both quantum groups we have (no sum on the $j$ )

$$
\psi_{m}^{j}\left(x_{1}^{\prime}, x_{2}^{\prime}\right)=\sum_{m^{\prime}} D_{m m^{\prime}}^{j} \psi_{m^{\prime}}^{j}\left(x_{1}, x_{2}\right)
$$

These representations are not strictly uniquely determined. They are only determined up to a $U(1)_{a} \times U(1)_{b}$ gauge transformation,

$$
a^{\prime}=e^{i \varphi_{a}} a, \quad b^{\prime}=e^{i \varphi_{b}} b, \quad c^{\prime}=e^{-i \varphi_{b}} c, \quad d^{\prime}=e^{-i \varphi_{a}} d
$$

which leaves the $a, b, c, d$ internal algebra unchanged. Explicitly, we have

$$
D_{m m^{\prime}}^{j}\left(q \mid a^{\prime}, b^{\prime}, c^{\prime}, d^{\prime}\right)=e^{i\left(\varphi_{a}+\varphi_{b}\right) m} e^{i\left(\varphi_{a}-\varphi_{b}\right) m^{\prime}} D_{m m^{\prime}}^{j}(q \mid a, b, c, d) .
$$

Unlike the undeformed case, there is a natural internal state space due to the fact that the $a, b, c, d$ can be thought of as noncommutative creation operators on an internal Fock space.

Finkelstein proposed an extension of the standard model based on the quantum group $S L_{q}(2)$ by replacing the elementary standard model field operators $\Psi(x)$ with $\hat{\Psi}_{m m^{\prime}}^{j} D_{m m^{\prime}}^{j}$ [7]. $S L_{q}(2)$ is the algebra of knots and $S U_{q}(2)$ is the algebra of oriented knots. Taking $N$ to be the crossing number knot invariant, $w$ to be the writhe of a knot, $r$ to be the rotation number of the two-dimensional projection of the corresponding classical three-dimensional knot and $o$ to be an odd number additional knot invariant required to preserve the difference in parity between the writhe and rotation, Finkelstein forms the identification

$$
\left(j, m, m^{\prime}\right)=\frac{1}{2}(N, w, r+o) \equiv \frac{1}{2}(N, w, \tilde{r})
$$

between the quantum state labels $\left(j, m, m^{\prime}\right)$ and the topological knot invariants $\frac{1}{2}(N, w, \tilde{r})$. The combined quantity $\tilde{r}$ is called the quantum rotation. Finkelstein identifies the elementary fermions $(e, \mu, \tau),\left(\nu_{e}, \nu_{\mu}, \nu_{\tau}\right),(u, c, t)$ and $(d, s, b)$ with the simplest nontrivial quantum knots, which are the $N=3$ quantum trefoil knots, of which there are four kinds represented by $D_{\frac{w}{2} \frac{r+1}{2}}^{N / 2}$ as $D_{\frac{3}{2} \frac{3}{2}}^{3 / 2}, D_{-\frac{3}{2} \frac{3}{2}}^{3 / 2}, D_{\frac{3}{2}-\frac{1}{2}}^{3 / 2}$ and $D_{-\frac{3}{2}-\frac{1}{2}}^{3 / 2}$. The first of these is identified with the $(e, \mu, \tau)$, the second with $\left(\nu_{e}, \nu_{\mu}, \nu_{\tau}\right)$, the third with $(d, s, b)$ and the fourth with $(u, c, t)$ elementary standard model fermions. The electroweak numbers isotopic spin $t$, third component $t_{3}$ and electroweak hypercharge $t_{0}$ are consistent with the empirical identification

$$
\left(j, m, m^{\prime}\right)=\frac{1}{2}(N, w, \tilde{r})=3\left(t,-t_{3},-t_{0}\right)_{L}
$$

for the left chiral fields and quantum trefoils. The trivial quantum knot case $N=1$ corresponds to twisted unknots which are naturally electroweak dark and hence can be identified as natural dark matter candidates. These so-called preon states nicely match up with the preons in the Harari and Shupe preon models [27, 28]. Since the representations are invariant only up to $U_{m} \times U_{m^{\prime}}$ transformations, and given that $m=\frac{w}{2}$ and $m^{\prime}=\frac{\tilde{r}}{2}$, there are Noether 
writhe and rotation charges $Q_{w}$ and $Q_{r}$ which are related to the electromagnetic charge $Q_{e}$ by $Q_{e} \equiv Q_{w}+Q_{r}$. In addition, one can write down $Q_{e}=e\left(t_{3}+t_{0}\right)=-\frac{e}{6}(w+\tilde{r})$. The electric charge then naturally provides a measure of the sum of writhe and quantum rotation of the trefoil.

Finkelstein interprets the noncommuting $a, b, c, d$ operator-valued elements of $D_{m m^{\prime}}^{j}$ to be fermionic preon creation operators with $a$ having charge $-\frac{e}{3}, d$ being its antiparticle and $b$ and $c$ being a neutral preon-antipreon pair. The preons correspond to twisted loops (as seen in their two-dimensional projections) and their quantum realizations $\hat{\Psi}_{m m^{\prime}}^{\frac{1}{2}} D_{m m^{\prime}}^{\frac{1}{2}}$ are interpreted as flux tubes. The knots have orientation and framing. The flow direction in the flux tube defines the helicity.

The electroweak vectors $W^{+}, W^{-}$and $W^{3}$, taking the $N=6$ representations, correspond to ditrefoil knots (which are knot sums of trefoil knots) and the electroweak quantum numbers work out analogously.

We have briefly reviewed the electroweak aspects of Finkelstein's knot model. We now briefly review the colour charge aspects and then a possible physical interpretation of the Hopf-type deformation parameter $q$. We have

$$
D_{\frac{3}{2} \frac{3}{2}}^{3 / 2} \approx a^{3}, \quad D_{-\frac{3}{2} \frac{3}{2}}^{3 / 2} \approx c^{3}, \quad D_{\frac{3}{2}-\frac{1}{2}}^{3 / 2} \approx a b^{2}, \quad D_{-\frac{3}{2}-\frac{1}{2}}^{3 / 2} \approx c d^{2}
$$

for the four classes of elementary leptons and for the $D_{-3 t_{3}-3 t_{0}}^{3}$ electroweak vector bosons $W^{+}, W^{-}$and $W^{3}$ we have respectively

$$
D_{-3,0}^{3} \approx c^{3} d^{3}, \quad D_{3,0}^{3} \approx a^{3} b^{3}, \quad D_{0,0}^{3} \approx A_{1} b^{3} c^{3}+A_{2} a b^{2} c^{2} d+A_{3} a^{2} b c d^{2}+A_{4} a^{3} d^{3}
$$

where the precise details of the coefficients $A_{i}$ do not concern us here. The important point here is that Finkelstein argues that in order to keep the Pauli exclusion principle we have to replace $(a, c)$ with $\left(a_{i}, c_{i}\right)$ where for charged leptons $a^{3} \rightarrow \epsilon^{i j k} a_{i} a_{j} a_{k}$ and for neutrinos $c^{3} \rightarrow \epsilon^{i j k} c_{i} c_{j} c_{k}$. The $a_{i}$ and $c_{i}$ yield a basis for the fundamental representation of $S U(3)$. Furthermore, if the $b$ preons and $d$ preons constitute colour singlets, it follows that $(d, s, b)$ type quarks $\left(a_{i} b^{2}\right)$ and $(u, c, t)$-type quarks $\left(c_{i} d^{2}\right)$ also provide a basis for the standard model fundamental representation of $S U(3)$. Finkelstein also points out that if one were to restrict to $S U_{q}(2)$, the algebra of oriented knots, where $\bar{a}=d$ and $\bar{c}=-q^{-1} b$, then $a_{i}$ and $c_{i}$ correspond to the $\overline{3}$-representation of $S U(3)$ while $b^{i}$ and $d^{i}$ correspond to the 3-representation of $S U(3)$. The electroweak vector bosons naturally are colour singlets in this model so these colour aspects of the model are internally consistent.

Regarding the physical interpretation of the Hopf-type deformation parameter $q$, Finkelstein arrives at the conjecture $q=\frac{e}{g}$ or $q=\frac{g}{e}$ with $e, g$ and $q$ being thought of as running coupling constants.

There are more interesting and important details in Finkelstein's knot model $S L_{q}(2)$ based extension to the standard model but we have now achieved our goal of describing the possible physical significance of the quantum groups $S L_{q}(2)$ and $S U_{q}(2)$ as describing knotted quantum flux tubes.

\section{References}

[1] H. Kodama, Prog. Theor. Phys. pp. 1024-1040 (1988)

[2] M. Gerstenhaber, The Annals of Mathematics 79, 59 (1964) 
[3] R.V. Mendes, Journal of Physics A: Mathematical and General 27, 8091 (1994)

[4] C. Chryssomalakos, E. Okon, Int.l J. Mod. Phys. D 13, 2003 (2004), [Arxiv:hepth/0407080]

[5] D. Ahluwalia-Khalilova, Class. Quantum Grav. 22, 1433 (2005)

[6] R. Finkelstein, Int. J. Mod. Phys. A pp. 4467-4480 (2007)

[7] R. Finkelstein, arXiv:1511.07919 (2015)

[8] R. Finkelstein, Int. J. Mod. Phys. A. pp. 4269-4302 (2006)

[9] R. Finkelstein, Int. J. Mod. Phys. A. pp. 6487-6494 (2005)

[10] R. Finkelstein, Int. J. Mod. Phys. A. pp. 627-650 (2003)

[11] R. Finkelstein, Lett. Math. Phys. pp. 157-163 (2000)

[12] R. Finkelstein, Mod. Phys. Lett. A pp. 1709-1716 (2000)

[13] R. Finkelstein, Lett. Math. Phys. pp. 105-114 (1999)

[14] S. Majid, A quantum groups primer. (Cambridge University Press, 2002)

[15] S. Majid, Foundations of quantum group theory. (Cambridge University Press, 1995)

[16] M. Bronstein, Physikalische Zeitschrift der Sowjetunion pp. 140-157 (1936)

[17] C. Rovelli, F. Vidotto, Covariant loop quantum gravity. (Cambridge University Press, 2002)

[18] B. Bertotti, Phys. Rev. pp. 1331-1333 (1959)

[19] I. Robinson, Bull. Acad. Polon. Sci. pp. 351-352 (1959)

[20] S. Majid, Proceedings of the International School of Physics: Quantum groups and their applications in physics pp. 267-345 (1994)

[21] E. Buffenoir, P. Roche, Commun. Math. Phys. pp. 669-698 (1995)

[22] E. Buffenoir, P. Roche, Commun. Math. Phys. (1999)

[23] K. Noui, P. Roche, Class. Quant. Grav. (2003)

[24] W. Fairbairn, C. Meusburger, J. Math. Phys. (2012)

[25] A. Randono, Ph.D. thesis, University of Texas at Austin Graduate School (2007)

[26] M. Frau, S. Sciuto, A. Lerda, Proceedings of the International School of Physics: Quantum groups and their applications in physics pp. 215-243 (1994)

[27] H. Harari, Phys. Lett. B 86, 83 (1979)

[28] M. Shupe, Phys. Lett. B. pp. 87-89 (1979) 